\title{
Prospective analysis of factors associated with inadequate bowel preparation for colonoscopy in actual clinical practice
}

\author{
Dae Hyung Woo, Kyeong Ok Kim, Da Eun Jeong, Yoon Jeong Nam, Si Hyung Lee, Byung Ik Jang, \\ Tae Nyeun Kim \\ Division of Gastroenterology and Hepatology, Department of Internal Medicine, Yeungnam University College of Medicine, Daegu, Korea
}

\begin{abstract}
Background/Aims: Inadequate bowel preparation can result in prolonged procedure time and increased missed lesion and complication rates. This prospective study aimed to evaluate bowel preparation quality and identify the predictive factors for inadequate bowel preparation in actual clinical practice. Methods: We included 399 patients who underwent colonoscopy between June 2015 and July 2016. Using the Aronchick bowel preparation scale, we defined a score $\leq 2$ as adequate preparation and a score $>2$ as inadequate preparation. Results: Mean patient age was $58.38 \pm 12.97$ years; $60.6 \%$ were male. Indications for colonoscopy included screening (69.7\%) and surveillance after polyp removal (21.3\%). A split-dose regimen was prescribed to $55.4 \%$ of patients. The inadequate bowel preparation rate was $28.1 \%$. Overall, the median time between the last bowel preparation agent dose and start of colonoscopy was 5.0 hours (range, 1.5-16.0 hours); that of the adequate group was 5.0 hours (range, 1.5-16.0 hours); and that of the inadequate group was 5 hours (range, 2-23 hours). The mean bowel preparation scale score of the ascending colon $(1.94 \pm 0.25)$ was significantly higher than that of other colon segments. On multivariate analysis, elderly age, history of cerebrovascular disease, history of gastrectomy or appendectomy, and total preparation solution uptake $<2 \mathrm{~L}$ were the independent predictors of inadequate bowel preparation. Conclusions: The inadequate bowel preparation rate was $28.1 \%$. Risk factors included elderly age and history of cerebrovascular disease or abdominal surgery. Patients with these risk factors require special care and education. (Intest Res 2018;16:293-298)
\end{abstract}

Key Words: Bowel preparation; Colonoscopy; Polyethylene glycols; Quality; Risk factors

\section{INTRODUCTION}

Screening colonoscopy (CFS) is widely performed in Korea as the incidence of colorectal cancer (CRC) continues to increase. ${ }^{1}$ Increasing the number of colonoscopies performed could reduce CRC incidence and later-stage diagnoses via the early detection and removal of precancerous lesions. However, CFS does not completely prevent all CRC. As interval CRC becomes an issue, CFS quality is emphasized.

The American Society for Gastrointestinal Endoscopy

Received July 20, 2017. Revised October 17, 2017.

Accepted October 20, 2017. Published online January 24, 2018

Correspondence to Kyeong Ok Kim, Division of Gastroenterology and

Hepatology, Department of Internal Medicine, Yeungnam University College of Medicine, 170 Hyeonchung-ro, Nam-gu, Daegu 42415, Korea. Tel: +82-

53-620-3830, Fax: +82-53-654-8386, E-mail: kokim@yu.ac.kr
(ASGE) and American Gastroenterological Association (AGA) proposed quality guidelines to indicate CFS performance. ${ }^{2}$ According to a recent study, these quality indicators were related to the occurrence of interval CRC. ${ }^{3}$ Among these CFS quality indicators, adequate bowel cleansing is among the most important factors. Inadequate bowel preparation is considered a common cause of CFS failure and can result in higher complication rates, a higher risk of procedure cancellation, increased cost and hospital stay length, extended procedure time, and lower detection of colon polyps. ${ }^{4,5}$

Although there are some suggestions about how bowel preparation may function as a quality indicator, ${ }^{2}$ there is no consensus of its ideal components. This prospective study aimed to evaluate the frequency of inadequate bowel preparation in actual clinical practice and identify its predictive factors prior to CFS.

( ) Copyright 2018. Korean Association for the Study of Intestinal Diseases. All rights reserved.

This is an Open Access article distributed under the terms of the Creative Commons Attribution Non-Commercial License (http://creativecommons.org/licenses/by-nc/4.0)

which permits unrestricted non-commercial use, distribution, and reproduction in any medium, provided the original work is properly cited. 


\section{METHODS}

\section{Subjects}

This single-center prospective study was performed between June 2015 and July 2016. Patients aged 18 to 80 years who underwent CFS at outpatient clinics with variable indications were prospectively and consecutively enrolled. We excluded cases of emergency CFS; patients with a history of colectomy, IBD, or familial adenomatous polyposis; and patients who were unable to provide informed consent. All colonoscopies were performed by 3 experienced endoscopists. Patient baseline characteristics such as sex, age, height, body weight, medication, waist circumference, history of abdominal surgery, other medical diagnoses, and current medications were collected before the scheduled CFS. Patient activity, bowel habits, time to CFS after preparation (hour), total amount of preparation solution uptake (L), pattern of last defecation before CFS, and mean procedure time (minute) were also assessed (Tables 1 and 2).

This study was approved by Institutional Review Board of Yeungnam University (YUMC 2015-04-002-001).

The patients provided written informed consent prior to participating.

\section{Bowel Preparation}

The patients were instructed by a nurse on how to prepare

Table 1. Baseline Patient Characteristics $(n=399)$

\begin{tabular}{ll}
\hline \multicolumn{1}{c}{ Variable } & Value \\
\hline Sex (male:female) & $1.65: 1$ \\
\hline Age $(\mathrm{yr})$ & $58.38 \pm 12.97$ \\
\hline BMl $\left(\mathrm{kg} / \mathrm{m}^{2}\right)$ & $23.36 \pm 3.03$ \\
\hline The aim of the procedure ${ }^{\mathrm{a}}$ & \\
\hline Screening & 278 \\
\hline Surveillance after polyp removal & 85 \\
\hline Constipation & 12 \\
\hline Diarrhea & 18 \\
\hline Others & 44 \\
\hline The presence of underlying disease & $168(42.1)$ \\
\hline History of operation & $102(25.6)$ \\
\hline
\end{tabular}

Values are presented as mean \pm SD or number (\%).

${ }^{a}$ The patients can have multiple aim of the procedure.

${ }^{b}$ History of colorectal cancer $(n=10)$, fecal occult blood $(n=10)$, abdomen pain $(n=10)$, weight loss $(n=7)$, anemia $(n=4)$, and family history of colorectal cancer $(n=3)$. and consume the bowel preparation solution with dietary restrictions before the examination using an introduction sheet. The patients were restricted to eat non-digestible foods such as vegetables, fruits, or grains for 3 days before the CFS. Patients were permitted only an early soft dinner the day before the CFS. Our patients received a low-volume (2L) polyethylene glycol solution with ascorbic acid (Coolprep; TaeJoon Pharmaceuticals, Seoul, Korea).

In the split bowel preparation protocol, the patient starts ingesting the first half of the bowel preparation solution ( $1 \mathrm{~L}$ ) at 18:00 the day before the examination and the second half of the preparation solution (1 L) starting at 06:00 the next day. In the single bowel preparation protocol, the patient begins to ingest the first half of the bowel preparation solution (1L) at 06:00; 2 to 3 hours later, they ingest the second half (1 L) over the course of 1 hour. Patients with morning CFS appointments tend to follow the split protocol, while those with an afternoon appointment tend to follow the single protocol. The time from the last ingestion to the CFS was measured for each patient.

\section{Assessment of Bowel Preparation}

The CFS procedures were performed by 3 endoscopists. After the CFS, each endoscopists reported their endoscopic exams and assessed the bowel preparation quality at 3 colon segments (right colon, transverse colon, left colon) using the Aronchick bowel preparation scale: ${ }^{5}$ "excellent, 1 point" (small volume of clear liquid or $>95 \%$ of surface visible), "good, 2 points" (large volume of clear liquid covering

Table 2. Characteristics of Colonoscopy Preparation Methods ( $n=399$ )

\begin{tabular}{lc}
\hline \multicolumn{1}{c}{ Variable } & Value \\
\hline Bowel cleaning regimen & $178(44.6) / 221(55.4)$ \\
Single/split & $290(72.7)$ \\
No low fiber diet within 48 hours of CFS & $3.03 \pm 0.54$ \\
Total amount of preparation solution uptake (L) & $3.06 \pm 0.49$ \\
Single & $3.01 \pm 0.61$ \\
Split & $5.0(1.5-16.0)$ \\
Time to CFS after preparation (hr) & $14(5-70)$ \\
Procedure time (min) & \\
Pattern of last defecation before colonoscopy & $387(97.0)$ \\
Clear water & $11(2.8)$ \\
Liquid with residue & $1(0.3)$ \\
\hline Semi-solid &
\end{tabular}

Values are presented as number (\%), mean \pm SD, or median (range). CFS, screening colonoscopy. 
$5 \%-25 \%$ of the surface but $>90 \%$ of surface visible), "fair, 3 points" (some semisolid stool that could be suctioned or washed away but $>90 \%$ of surface visible), "poor, 4 points" (semisolid stool that could not be suctioned or washed away and $<90 \%$ of surface visible), "inadequate, 5 points" (solid stool that impedes the vision for which a repeat preparation and CFS are needed). For the purposes of the analysis, we combined patients with excellent and good preparations into an adequate category and patients with fair, poor, or inadequate preparations into an inadequate category. We then calculated the average score of 3 parts of the colon and defined a score $\leq 2$ as adequate preparation (A group) and a score $>2$ as inadequate preparation (I group). All exams were recorded onto a picture archiving and communication system.

\section{Statistical Analysis}

The required number of subjects was calculated using the PS program (http://biostat.mc.vanderbilt.edu/wiki/Main/ PowerSampleSize) with $80 \%$ power and 5\% significance probability. All analyses were conducted using SPSS version
18.0 (SPSS Inc., Chicago, IL, USA). Values are expressed as mean \pm SD. Distributions of continuous variables were analyzed by Pearson correlation and one-way ANOVA or Student $t$-test. Comparisons of categorical variables were performed by the chi-square test or Fisher exact test. Variables revealed to be significant predictors on univariate analysis were assessed using multivariate logistic regression modeling. $P$-values $<0.05$ were considered statistically significant.

\section{RESULTS}

A total of 399 consecutive patients were enrolled. Mean patient age was $58.38 \pm 12.97$ years and $60.6 \%$ were male. Their mean BMI was $23.36 \pm 3.03 \mathrm{~kg} / \mathrm{m}^{2}$. Indications for CFS were $69.7 \%$ screening, $21.3 \%$ surveillance after polyp remov$\mathrm{al}$, and $3.0 \%$ constipation. Just below half $(42.1 \%)$ of the patients in our study revealed having at least one comorbidity such as hypertension or cerebrovascular disease, and onequarter $(25.6 \%)$ of them had a history of abdominal surgery (Table 1). The last stool status before CFS was clear liquid in 387 patients $(96.9 \%)$.

Just over half ( $\mathrm{n}=221,55.4 \%)$ of our patients followed the

Table 3. Clinical Comparison of Patients by Adequate or Inadequate Bowel Preparation Findings

\begin{tabular}{|c|c|c|c|}
\hline Risk factor & Adequate preparation $(n=287)$ & Inadequate preparation $(n=112)$ & $P$-value \\
\hline Age (yr) & $57.19 \pm 13.46$ & $61.41 \pm 11.10$ & 0.003 \\
\hline Male sex & $174(60.6)$ & 71 (63.4) & 0.610 \\
\hline BMI $\left(\mathrm{kg} / \mathrm{m}^{2}\right)$ & $23.41 \pm 3.63$ & $23.23 \pm 3.02$ & 0.452 \\
\hline Comorbidity & $112(39.0)$ & $56(50.0)$ & 0.046 \\
\hline Constipation & $8(2.8)$ & $4(3.6)$ & 0.680 \\
\hline Diabetic mellitus & $37(12.9)$ & $22(19.6)$ & 0.088 \\
\hline Hypertension & $74(25.8)$ & $31(27.7)$ & 0.699 \\
\hline Liver disease & $8(2.8)$ & $5(4.5)$ & 0.397 \\
\hline IBD & $9(3.1)$ & $5(4.5)$ & 0.517 \\
\hline Cardiovascular disease & $20(7.0)$ & $10(8.9)$ & 0.505 \\
\hline Cerebrovascular disease & $3(1.0)$ & $6(5.4)$ & 0.009 \\
\hline History of operation & $57(19.9)$ & $45(40.2)$ & 0.000 \\
\hline Appendectomy & $19(6.6)$ & $17(15.2)$ & 0.007 \\
\hline Gastrectomy & $21(7.3)$ & $22(19.6)$ & 0.000 \\
\hline Cholecystectomy & $11(3.8)$ & $7(6.2)$ & 0.296 \\
\hline Split regimen & $166(75.0)$ & $55(25.0)$ & 0.115 \\
\hline Mean amount of preparation solution uptake (L) & $3.06 \pm 0.59$ & $2.95 \pm 0.51$ & 0.387 \\
\hline Low solution uptake ( $\leq 2 \mathrm{~L}$ ) & $14(4.9)$ & $15(13.4)$ & 0.003 \\
\hline No. of defecation & $8.90 \pm 2.74$ & $9.11 \pm 4.12$ & 0.001 \\
\hline Procedure in the morning/afternoon & $173(60.3) / 114(39.7)$ & $53(47.3) / 59(52.7)$ & 0.019 \\
\hline
\end{tabular}

Values are presented as mean \pm SD or number (\%). 
Table 4. Risk Factors of Poor Bowel Preparation by Multivariate Analysis

\begin{tabular}{lccc}
\hline \multicolumn{1}{c}{ Risk factor } & Adjusted OR & \multicolumn{1}{c}{$\mathbf{9 5 \%}$ Cl } & $\boldsymbol{P}$-value \\
\hline Age & 1.02 & $1.003-1.042$ & 0.026 \\
Cerebrovascular disease & 6.53 & $1.542-27.635$ & 0.011 \\
History of gastrectomy & 3.21 & $1.616-6.369$ & 0.001 \\
History of appendectomy & 2.93 & $1.412-6.093$ & 0.004 \\
Low solution uptake $\leq 2 \mathrm{~L}$ & 3.60 & $1.598-8.114$ & 0.002 \\
\hline
\end{tabular}

split protocol, while the others $(\mathrm{n}=178,44.6 \%)$ followed the single protocol. Regarding colon preparation quality, 104 of colonoscopies (26.1\%) were judged as "excellent," 183 (45.8\%) as "good," 99 (24.8\%) as "fair," 12 (3.0\%) as "poor", and $1(0.1 \%)$ as "inadequate." Overall, 287 patients (71.9\%) achieved adequate bowel preparation and 112 patients (28.1\%) achieved inadequate bowel preparation. Overall, the median time between the last dose of the bowel preparation agent and the start of CFS was 5.0 hours (1.5-16.0 hours); that in the A group was 5.0 hours (1.5-16.0 hours); and that in the I group was 5 hours ( $2-23$ hours $)(P=0.190)$. The overall median procedure time was 14 minutes (5-70 minutes); that in the A group was 15 minutes (5-60 minutes); and that in the I group was 17.5 minutes $(5-70$ minutes $)(P=0.950)$ (Table 2).

CFS was performed in the afternoon in $39.7 \%(n=114)$ of patients in the A group versus $52.7 \%(\mathrm{n}=59)$ of patients in the I group $(P=0.02)$. Patients who underwent abdominal surgery were more in I group $(19.9 \%$ vs. $40.2 \%, P<0.01)$. A total preparation solution uptake of $<2 \mathrm{~L}$ was reported by $4.9 \%$ of patients $(\mathrm{n}=14)$ in the A group versus $13.4 \%$ of patients $(\mathrm{n}=15)$ in the I group $(P<0.01)$ (Table 3$)$.

On multivariate analysis, elderly age (OR, 1.02; 95\% CI, 1.003-1.042; $P=0.03$ ), history of cerebrovascular disease (OR, 6.53; 95\% CI, 1.542-27.635; $P<0.01$ ), history of gastrectomy (OR, 3.21; 95\% CI, 1.616-6.369; $P<0.01$ ) or appendectomy (OR, 2.93; 95\% CI, 1.412-6.093; $P<0.01$ ), and a total intake of the preparation solution of $<2 \mathrm{~L}(\mathrm{OR}, 3.60 ; 95 \% \mathrm{CI}$, 1.598-8.114; $P<0.01)$ were the independent predictors of inadequate bowel preparation (Table 4 ).

\section{DISCUSSION}

Inadequate bowel preparation results in a reduced adenoma detection rate and is associated with interval CRC. ${ }^{6}$ According to the ASGE and AGA suggestions of CFS quality indicators, adequate preparation should be $>98 \%{ }^{2}$ To accomplish this target rate, it is very important to identify patients with risk factors of poor bowel preparation to enable improvement. Accordingly, we aimed to investigate the frequency of inadequate bowel preparation in real clinical practice and the associated risk factors that need to be improved.

Previous studies reported that the inadequate bowel preparation rate was $21 \%$ to $28 \%$, similar to our findings. Our results showed the status of actual clinical practice, and considering the suggested proportion of adequate bowel preparation in quality indicator of CFS, ${ }^{2}$ further stress the need for greater attention on improving bowel preparation status.

Our study findings suggested that elderly age, history of cerebrovascular disease, history of gastrectomy or appendectomy, and a total preparation solution uptake $<2 \mathrm{~L}$ are significant predictors of inadequate bowel preparation. These results were similar to those of several previous studies. $^{1,-9}$

As life expectancy increases, the indication of CFS in elderly patients also increases. ${ }^{10}$ One study showed that the incidence of inadequate preparation in old age is high. ${ }^{11}$ In the current study, increased age was an independent risk factor for inadequate bowel preparation, which could be associated with delayed gastrointestinal motility, poor understanding of preparation instructions, high constipation rate, comorbid diseases such as diabetes, and a higher incidence of a history of abdominal surgery.

Chung et al. ${ }^{1}$ reported that a history of colectomy, hysterectomy, or appendectomy was a risk factor of inadequate bowel preparation. However, our data did not reveal any difference in bowel preparation status by history of hysterectomy, likely because too few patients had a history of hysterectomy $(n=6)$. We found that a history of other intra-abdominal surgeries such as appendectomy and gastrectomy were independent risk factors of inadequate bowel preparation. These findings suggest that any kind of intra-abdominal surgery could affect bowel preparation status due to altered bowel motility and postoperative adhesions.

Failure to ingest sufficient preparation solution is also a cause of inadequate bowel preparation. ${ }^{12-15}$ Although several kinds of preparation solutions have become available in an effort to improve compliance, patient satisfaction is limited. Common reasons for noncompliance include patient discomfort and intolerance to ingesting a large solution volume. ${ }^{16}$ In our study, 29 patients (7.3\%) ingested an inadequate preparation solution volume $(<2 \mathrm{~L})$. Although Chiu et al. ${ }^{17}$ reported that the ingestion of $2 \mathrm{~L}$ of bowel preparation solution was sufficient to achieve an adequate bowel preparation, if ingested according to the preparation time protocol, 
doing so in actual practice is not so easy for patients.

In our study, a history of cerebrovascular disease was an independent predictor of inadequate bowel preparation. The possible causes for this include an increased incidence of bed-ridden status, a high frequency of constipation, delayed gastrointestinal motility, and medications that could affect the bowel motility. ${ }^{9,18,19}$

A previous study suggested other factors such as medical problems that influence bowel motility including liver cirrhosis, diabetes, chronic constipation, multiple medications, obesity, and hospitalization as predictors of inadequate bowel preparation. ${ }^{1}$ However, the current study did not show any significant results regarding these factors, possibly due to inadequate numbers of patients with these comorbidities.

In a recent study, the split regimen was reportedly more effective than the single regimen for achieving dose in bowel preparation; ${ }^{20,21}$ however, our study revealed no significant intergroup difference. This may be due to 15 of 221 patients (6.9\%) who received a split dose versus 3 of 178 patients (1.7\%) who received the single dose vomiting during the bowel preparation process, representing a statistically significant difference $(P=0.01)$ and possibly offsetting the benefit of the split dose. In addition, our study intended to reveal the actual practice, in which patient characteristics could be heterogeneous and preparation regimen may be of no significance.

In our study, patients who underwent CFS in the afternoon had a higher incidence of inadequate bowel preparation (A group, 114 [39.7\%]; I group, 59 [52.7\%]; $P=0.02$ ) as well as a higher incidence of being prescribed the single bowel preparation protocol. However, inadequate bowel preparation was not significantly associated with single bowel preparation protocol. Inadequate bowel preparations were more frequent in the afternoon patients because these patients had a higher incidence of a history of cerebrovascular disease or intra-abdominal surgery (morning patients, 47 [20.8\%]; afternoon patients, $63[36.4 \%])$. These results suggest that other factors as well as compliance are more influential than regimen method.

In our study, $72.7 \%$ of patients ingested a low fiber diet for the 48 hours prior to CFS, while $92 \%$ ingested a total uptake preparation solution volume $>2$ L. A low understanding of the instructions and poor awareness of the results of inadequate bowel preparation could result in patient noncompliance. Nguyen and Wieland ${ }^{22}$ reported that a poor understanding of the instructions is a significant factor of poor bowel preparation. Education regarding bowel preparation could effectively improve bowel preparation status. ${ }^{23-25}$ Lee et $\mathrm{al}^{26}$ also reported that bowel preparation protocol reinforcement education by telephone or text message increased the adequate bowel preparation rate. Using these methods, especially with elderly patients, will help increase the adequate bowel preparation rate.

Our study has several limitations. First, the bowel preparation status assessments were performed by 3 endoscopists, which may introduce inter-observer variation. To reduce it, the endoscopists referred to a photographic example of bowel preparation conditions at every examination prior to scoring. Second, because the data of total solution intake, number of bowel movements, time to CFS after preparation, and pattern of last defecation before CFS depended on patient memory, recall bias could be a factor. Third, to see the actual clinical outcomes, patients who were excluded from other studies (those with a history of surgery or other factors) were included in our study, which could create a confounding factor.

Nevertheless, in Asia, our study included relatively large numbers of patients who were subjected to a standard CFS protocol. As such, it can reflect the degree of bowel preparation in actual clinical practice. Our results may help clinicians identify patients at high risk of inadequate bowel preparation.

In conclusion, $28.1 \%$ of our patients had inadequate bowel preparation, the risk factors of which were elderly age, a history of intra-abdominal surgery such as appendectomy or gastrectomy, a history of cerebrovascular disease, and inadequate preparation solution consumption( $<2 \mathrm{~L})$. Our findings suggest that special care and an individualized approach are required for patients with risk factors of inadequate bowel preparation, including a comprehensive education program about preparation and the encouragement of an appropriate diet and ambulation during preparation for CFS.

\section{FINANCIAL SUPPORT}

The authors received no financial support for the research, authorship, and/or publication of this article.

\section{CONFLICT OF INTEREST}

No potential conflict of interest relevant to this article was reported.

\section{AUTHOR CONTRIBUTION}

Dae Hyung Woo: data analysis, make draft 
Kyeong Ok Kim: colonoscopy, design, data analysis, revision

Da Eun Jeong: data review, revision

Yun Jeong Nam: design, data analysis, Data review

Byung Ik Jang, Si Hyung Lee: colonoscopy, revision

Tae Nyeun Kim: design, revision

\section{REFERENCES}

1. Chung YW, Han DS, Park KH, et al. Patient factors predictive of inadequate bowel preparation using polyethylene glycol: a prospective study in Korea. J Clin Gastroenterol 2009;43:448-452.

2. Rex DK, Schoenfeld PS, Cohen J, et al. Quality indicators for colonoscopy. Am J Gastroenterol 2015;110:72-90.

3. Adler J, Robertson DJ. Interval colorectal cancer after colonoscopy: exploring explanations and solutions. Am J Gastroenterol 2015;110:1657-1664.

4. Harewood GC, Sharma VK, de Garmo P. Impact of colonoscopy preparation quality on detection of suspected colonic neoplasia. Gastrointest Endosc 2003;58:76-79.

5. Aronchick CA. Bowel preparation scale. Gastrointest Endosc 2004;60:1037-1038.

6. Chokshi RV, Hovis CE, Hollander T, Early DS, Wang JS. Prevalence of missed adenomas in patients with inadequate bowel preparation on screening colonoscopy. Gastrointest Endosc 2012;75:1197-1203.

7. Cheng RW, Chiu YC, Wu KL, et al. Predictive factors for inadequate colon preparation before colonoscopy. Tech Coloproctol 2015;19:111-115.

8. Hautefeuille G, Lapuelle J, Chaussade S, et al. Factors related to bowel cleansing failure before colonoscopy: results of the PACOME study. United European Gastroenterol J 2014;2:22-29.

9. Ness RM, Manam R, Hoen H, Chalasani N. Predictors of inadequate bowel preparation for colonoscopy. Am J Gastroenterol 2001;96:1797-1802.

10. Kim HG. Is elderly age a simple predictive factor for inadequate bowel preparation before colonoscopy? Gut Liver 2016;10:489490.

11. Clark BT, Laine L. High-quality bowel preparation is required for detection of sessile serrated polyps. Clin Gastroenterol Hepatol 2016;14:1155-1162.

12. Marshall JB, Pineda JJ, Barthel JS, King PD. Prospective, randomized trial comparing sodium phosphate solution with polyethylene glycol-electrolyte lavage for colonoscopy preparation. Gastrointest Endosc 1993;39:631-634.

13. Kolts BE, Lyles WE, Achem SR, Burton L, Geller AJ, MacMath T. A comparison of the effectiveness and patient tolerance of oral sodium phosphate, castor oil, and standard electrolyte lavage for colonoscopy or sigmoidoscopy preparation. Am J Gastroenterol 1993;88:1218-1223.
14. Cohen SM, Wexner SD, Binderow SR, et al. Prospective, randomized, endoscopic-blinded trial comparing precolonoscopy bowel cleansing methods. Dis Colon Rectum 1994;37:689-696.

15. Afridi SA, Barthel JS, King PD, Pineda JJ, Marshall JB. Prospective, randomized trial comparing a new sodium phosphatebisacodyl regimen with conventional PEG-ES lavage for outpatient colonoscopy preparation. Gastrointest Endosc 1995;41:485-489.

16. Hsu CW, Imperiale TF. Meta-analysis and cost comparison of polyethylene glycol lavage versus sodium phosphate for colonoscopy preparation. Gastrointest Endosc 1998;48:276-282.

17. Chiu HM, Lin JT, Wang HP, Lee YC, Wu MS. The impact of colon preparation timing on colonoscopic detection of colorectal neoplasms: a prospective endoscopist-blinded randomized trial. Am J Gastroenterol 2006;101:2719-2725.

18. Bassotti G, Gaburri M, Imbimbo BP, et al. Colonic mass movements in idiopathic chronic constipation. Gut 1988;29:11731179.

19. Hardcastle JD, Mann CV. Study of large bowel peristalsis. Gut 1968;9:512-520.

20. Horton N, Garber A, Hasson H, Lopez R, Burke CA. Impact of single- vs. split-dose low-volume bowel preparations on bowel movement kinetics, patient inconvenience, and polyp detection: a prospective trial. Am J Gastroenterol 2016;111:13301337.

21. ASGE Standards of Practice Committee, Saltzman JR, Cash BD, et al. Bowel preparation before colonoscopy. Gastrointest Endosc 2015;81:781-794.

22. Nguyen DL, Wieland M. Risk factors predictive of poor quality preparation during average risk colonoscopy screening: the importance of health literacy. J Gastrointestin Liver Dis 2010;19:369-372.

23. Abuksis G, Mor M, Segal N, et al. A patient education program is cost-effective for preventing failure of endoscopic procedures in a gastroenterology department. Am J Gastroenterol 2001;96:1786-1790.

24. Rosenfeld G, Krygier D, Enns RA, Singham J, Wiesinger H, Bressler B. The impact of patient education on the quality of inpatient bowel preparation for colonoscopy. Can J Gastroenterol 2010;24:543-546.

25. Rodger J, Steele RJ. Telephone assessment increases uptake of colonoscopy in a FOBT colorectal cancer-screening programme. J Med Screen 2008;15:105-107.

26. Lee YJ, Kim ES, Choi JH, et al. Impact of reinforced education by telephone and short message service on the quality of bowel preparation: a randomized controlled study. Endoscopy 2015;47:1018-1027. 